\title{
Coronavirus Transcription: A Perspective
}

\author{
S. G. Sawicki $(\bullet) \cdot$ D. L. Sawicki
}

Department of Microbiology, Medical College of Ohio, Toledo, OH 43614, USA ssawicki@mco.edu

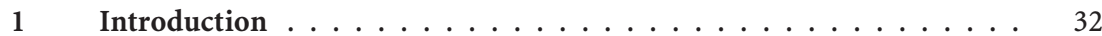

2 Discontinuous Transcription by Coronaviruses. . . . . . . . . . 35

3 Kinetics of Plus- and Minus-Strand RNA Synthesis and Sensitivity to Translational Inhibition . . . . . . . . . . 41

4 The Kinetics of Synthesis of the Subgenomic Minus Strands . . . . . 42

$5 \quad$ Subgenomic MHV RIs Exist in Infected Cells and Are Transcriptionally Active in mRNA 2-7 Synthesis . . . . . . 43

6 Characterization of Coronavirus Native RI/TIs and Native RF/TFs . . . 44

7 Turnover of MHV Replicative/Transcriptive Intermediates . . . . . . 48

$8 \quad$ A Working Model . . . . . . . . . . . . . . . . . . . . . . 50

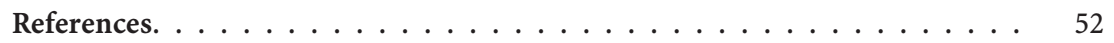

Abstract At the VIth International Symposium on Corona and Related Viruses held in Québec, Canada in 1994 we presented a new model for coronavirus transcription to explain how subgenome-length minus strands, which are used as templates for the synthesis of subgenomic mRNAs, might arise by a process involving discontinuous RNA synthesis. The old model explaining subgenomic mRNA synthesis, which was called leader-primed transcription, was based on erroneous evidence that only genome-length negative strands were present in replicative intermediates. To explain the discovery of subgenome-length minus strands, a related model, called the replicon model, was proposed: The subgenomic mRNAs would be produced initially by leader-primed transcription and then replicated into minus-strand templates that would in turn be transcribed into subgenomic mRNAs. We review the experimental evidence that led us to formulate a third model proposing that the discontinuous event in coronavirus RNA synthesis occurs during minus strand synthesis. With our model the genome is copied both continuously to produce minus-strand templates for genome RNA synthesis and discontinuously to produce minus-strand templates for subgenomic mRNA synthesis, and the subgenomic mRNAs do not function as templates for minus strand synthesis, only the genome does. 


\section{1}

\section{Introduction}

Our studies focus on a Group 2 coronavirus, mouse hepatitis virus strain A59 (MHV-A59). MHV-A59 was isolated in 1961 from a colony of $\mathrm{Balb} / \mathrm{C}$ mice that were being used to serially propagate leukemia that was caused by a virus (Manaker et al. 1961; David-Ferreira and Manaker 1965). Probably multiple serial passages of cell-free filtrates of leukemic cells in newborn mice selected from an endogenous mouse coronavirus a variant that replicated to high titer quickly and that produced, as a consequence, hepato-encephalopathy. In our laboratory MHV-A59 has the characteristic of replicating to high titer $\left(>10^{9} \mathrm{pfu} / \mathrm{ml}\right)$ when cells such as 17cl-1 cells that express mCEACAM, e.g., the receptor for MHV, are used. On 17cl-1 cells, which were derived from spontaneously transformed Balb/C 3T3 fibroblasts (Sturman and Takemoto 1972), MHVA59 causes cell fusion, forms very efficiently clear plaques of $\sim 5-\mathrm{mm}$ diameter after 2 days, and produces about $10^{4}$ virions per cell and $3 \times 10^{3} \mathrm{pfu} / \mathrm{cell}$ within $8 \mathrm{~h}$ (our unpublished observations). This makes MHV-A59 an ideal virus to study its replication at the molecular and genetic level. Essentially all of our studies used MHV-A59 and 17cl-1 cells.

Like all coronaviruses, MHV-A59 possesses a large $(31.4 \mathrm{~kb}$ for $\mathrm{MHV}$ A59), single-stranded, messenger- or plus-sense RNA genome that contains a poly $(\mathrm{A})$ sequence at the $3^{\prime}$ end and a methylated guanosine cap at the $5^{\prime}$ end, although the length of the poly(A) sequence and the cap structure have not been extensively characterized. The genome is associated with a helical nucleocapsid composed of the $\mathrm{N}$ protein of $50-$ $60 \mathrm{kDa}$. Surrounding the nucleocapsid is a membrane in which is embedded the envelope or spike (S) glycoprotein of $180 \mathrm{kDa}$, which may be cleaved in half by a host protease and which produces characteristic surface projections on the virions. Also embedded in the membrane are two other envelope proteins: a multi-spanning membrane (M) glycoprotein of $23 \mathrm{kDa}$ essential for the assembly of virions and an E protein of $10 \mathrm{kDa}$, which although not absolutely essential for the formation of virions, is required for the release of large numbers of virions from infected cells (Kuo and Masters 2003). In addition, some strains of MHV produce virions containing an HE glycoprotein and an I protein, neither of which is essential for viral infectivity or growth. The virions form internally, accumulate in vesicles in the cytoplasm, and are released rapidly en masse by exocytosis. Recent publications (Siddell 1995; Lai and Cavanagh 1997; Lai and Holmes 2001) review and cite extensive literature on coronaviruses. 
Presumably, a single molecule of genome RNA is capable of initiating a successful round of viral replication in the cytoplasm without the need of nuclear functions: MHV replicates in dactinomycin-pretreated cells. Viral structural proteins are not required for viral RNA synthesis because purified, deproteinized genomes obtained from virions are infectious. More recently it was shown that an infectious clone of the human coronavirus $229 \mathrm{E}$ with the genes encoded by the subgenomic mRNAs, including the structural genes for S, E, M, and N, deleted was able to replicate and produce a subgenomic mRNA encoding a nonviral fluorescent protein (Thiel et al. 2001a,b). Therefore, the proteins produced by the subgenomic mRNAs probably do not function in viral RNA synthesis. Further evidence of this is that the ratio of the synthesis of genome to subgenomic mRNA does not change from the earliest times postinfection (p.i.) to very late times p.i., even after virion production has ended and viral RNA synthesis is declining (our unpublished observations). Therefore, the accumulation of structural proteins such as $\mathrm{N}$ (nucleocapsid proteins) does not affect or change the transcriptional pattern, that is, the ratio of the synthesis of genome to subgenomic mRNA.

What makes coronaviruses particularly interesting is that they have the largest genome of the RNA viruses, which encodes a large number of gene products that are unique to viruses belonging to the Nidovirales, and they utilize a discontinuous RNA synthetic process to produce subgenomic messenger RNA molecules. During replication of MHV-A59, genomic (RNA-1) and six subgenomic mRNAs (RNA-2 of $9.6 \mathrm{~kb}$, RNA-3 of $7.4 \mathrm{~kb}, \mathrm{RNA}-4$ of $3.4 \mathrm{~kb}$, RNA-5 of $3.0 \mathrm{~kb}$, RNA-6 of $2.4 \mathrm{~kb}$, RNA-7 of $1.7 \mathrm{~kb})$ are produced. These together comprise a $3^{\prime}$ coterminal nested set (Fig. 1). The mRNA species vary in size from one-third (mRNA 2) to about one-twentieth (mRNA 7) of the genome. For MHV-A59 plus strands (genomes and subgenomic mRNA) are produced in large amounts and, at about $1 \%$ of this number, minus strands of both genome- and subgenomic length that serve as the templates for genome and subgenomic mRNA synthesis (Sawicki and Sawicki 1986; Sawicki and Sawicki 1990).

On infection the first open reading frame (ORF-1) in the genome of MHV-A59, which is $~ 20,000 \mathrm{nt}$ and divided into Repla and Rep1b by a ribosomal frameshift, is translated as two large polyproteins, ppla and pplab (Fig. 2). Three functional proteinases (PRO), two papainlike (PL1 and PL2) and one poliovirus 3C-like (3CL), are located in ppla and cleave the ppla and pp1ab into 16 nonstructural proteins, nsp1-16 (for relevant references see Brockway et al, 2003). These proteins and/or the polyprotein precursors form the viral RNA-dependent RNA polymerase. 


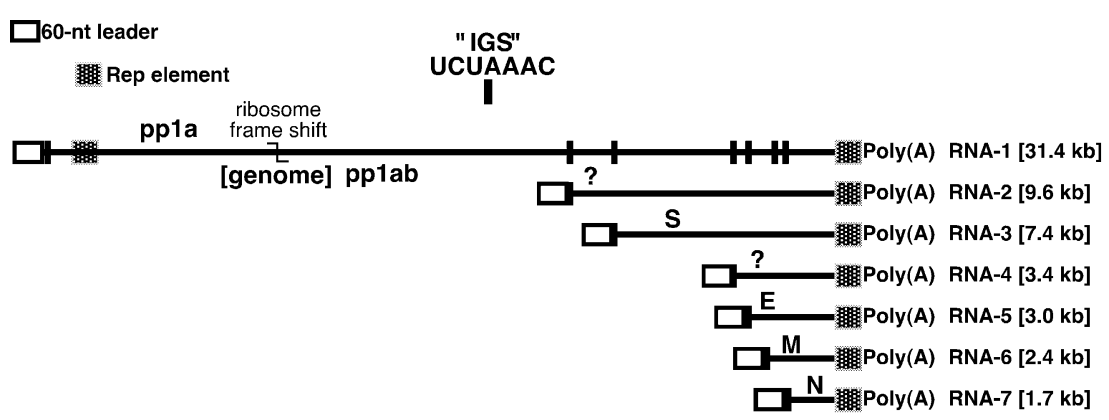

Fig. 1. The coronavirus genome RNA and six subgenomic mRNAs share identical $3^{\prime}$ sequences and form a $3^{\prime}$ nested set of RNAs. The genome is translated into two large polyproteins that are encoded in the large ORF 1 sequence. Only the ORF at the $5^{\prime}$ region of each of the subgenomic mRNAs is translated into a unique protein, making the genome and subgenomic mRNAs functionally monogenic

Very quickly after entry MHV-A59 produces first the minus-strand templates for genome and subgenomic mRNA synthesis and then the transcription complexes that transcribe the minus-strand templates into genomes and subgenomic mRNAs. Recently Alexander Gorbalenya and his colleagues (Snijder et al. 2003) described the actual and potential functions of the proteins encoded in ORF-1 of the SARS-CoV and related coronaviruses and members of the order Nidovirales. The question im-

\section{ORF-1}

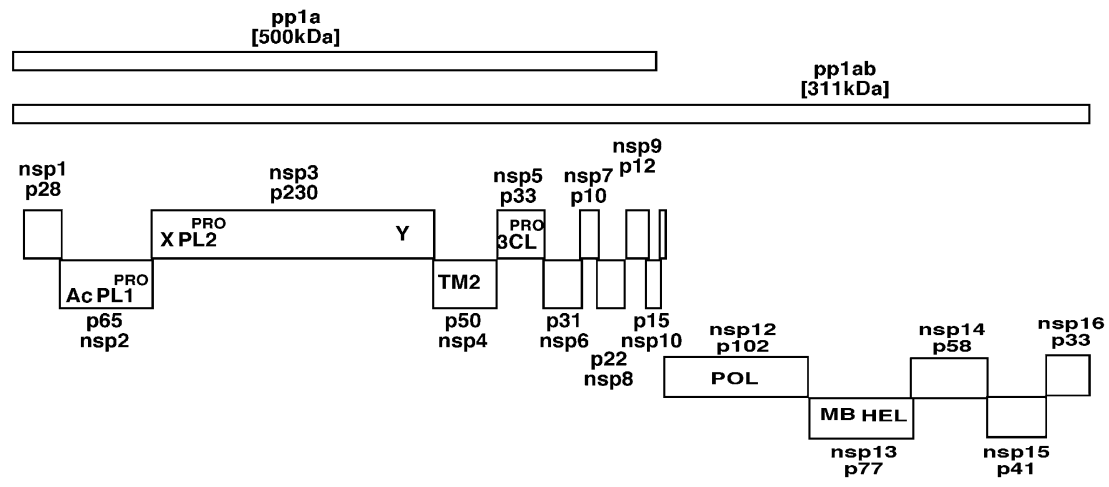

Fig. 2. A schematic showing the ORF1 of the coronavirus genome and its ppla and pplab polyproteins and processed products. See Snijder et al. (2003) for detailed information 
mediately arises as to why Nidoviruses possess so many individual proteins, especially where other plus-strand viruses make do with 1-4 proteins for viral RNA synthesis and one-half to one quarter the number of amino acids. These extra proteins could be needed to produce a unique replication and transcription machinery, to increase the fidelity of RNAdependent RNA synthesis that is inherently error prone, to replace needed host factors that are required by other viruses, or to interact with and modulate the host cell or the immune system of the host animal. If mutations in all of the 15-16 genes of MHV give phenotypes that affect viral RNA synthesis, it would suggest that Nidoviruses utilize fundamentally different replication/transcription machinery than other plus-strand viruses. The viral gene products would also interact with those of the host cell because it is likely that the host cell provides functions not encoded by the viral genes.

\section{2 \\ Discontinuous Transcription by Coronaviruses}

Whereas genome-sized plus and minus strands are made by the viral replicases continuously or processively copying their respective templates, discontinuous transcription is the mechanism responsible for generating the minus-strand templates for the subgenomic mRNA. The first evidence for discontinuous synthesis was the discovery that all of the viral plus strands possessed the 1.7-kb sequence of RNA-7, in addition to the poly(A) tract at their $3^{\prime}$ ends, and at their $5^{\prime}$ ends, the genome and all subgenomic mRNAs possessed an identical "leader" sequence of $\sim 60$ nt (Spaan et al. 1983). It was realized that because this leader sequence is restricted to the $5^{\prime}$ end of the genome, viral RNA synthesis must include a process by which the leader RNA is joined to the "body" of mRNAs 2-7 that are located at the $3^{\prime}$ end of the genome. At the end of the leader and before the body of each open reading frame of the subgenomic mRNAs is an "intergenic sequence," or "IGS or IG sequence" or "TRS," or "transcription regulating sequence" (van der Most et al. 1994; van Marle et al. 1995). Some also call it the "TAS", or "transcription activation sequence." For MHV-59 the IGS is ${ }^{{ }^{\prime}}$ UCUAAAC $^{3^{\prime}}$. The same replication strategy appears in the Arteriviridae that recently have been united with the Coronaviridae within the order Nidovirales (Cavanagh 1997).

Lai and his collaborators gave a stimulus to research on coronaviruses when they produced a model to explain the generation of subgenomic 
mRNAs of coronaviruses (Baric et al. 1983). They suggested that the viral polymerase (RdRp) initiated plus strand synthesis at the $3^{\prime}$ end of genome-length templates. In this model, after it produced the leader RNA and the IGS, the RdRp would jump from the $3^{\prime}$ end of the minus strand all the way to one of the internal complementary copies of the IGS and reinitiate RNA synthesis. The IGS served to redirect the RdRp to an internal site and then served as a primer for elongation. This "leaderprimed transcription model" was based on three experimental findings. First, subgenomic mRNAs comprised a nested set of $3^{\prime}$ coterminal RNA molecules and each contained a $5^{\prime}$ leader sequence that was present only once at the $5^{\prime}$ end of the genome (Lai et al. 1983; Spaan et al. 1983; Lai et al. 1984). Second, formation of subgenomic RNAs by splicing was ruled out by finding that the UV light sensitivity for the synthesis of each species of viral plus-strand RNA was directly proportional to the RNA length (Jacobs et al. 1981; Stern and Sefton 1982). Third, cells infected with MHV were reported to contain only a full-length minus-strand copy of the genome; no subgenomic minus-strand RNA was detectable (Lai et al. 1982). They strengthened their argument by showing that only replication intermediates (RIs) with genome-length templates could be found in infected cells and that treatment of the RIs with ribonuclease (RNase) produced only RF RNA of genome length. The only model that could account for or explain these observations was their "leaderprimed" model.

In the years following the proposal of the leader-primed transcription model it became clear that this model did not explain the newer experimental findings. Some of these experimental results directly invalidated the leader-primed transcription model or showed that experimental conditions used for detection of subgenomic minus-strand RNA were not optimal because of excessive digestion of the RNA complexes with RNase A or the ${ }^{32}$ P-labeled RNA probes used (Lai et al. 1982: Baric et al. 1983) were likely of too low a specific activity to detect minus strands (Sawicki et al. 2001).

David Brian's lab (Sethna et al. 1989) showed that cells infected with a coronavirus (porcine transmissible gastroenteritis virus, TGEV) contained a $5^{\prime}$ nested set of minus strands that corresponded in size to the genome and the subgenomic mRNA. They did this by Northern blotting, using in vitro labeled probes to the minus and plus strands. After seeing their publication, we thought it was possible that the subgenome-length minus strands they observed were created during preparation of the infected cell lysates. They might have arisen from genome-length minus strands in RIs. Activation of endogenous RNase might have cleaved the 
native, genome-length RI molecules into subgenomic RFs by targeting certain nuclease-sensitive sites in the genome template. This occurs with alphavirus RIs, whose genome-length template can be cleaved specifically by RNase at a single, internal site that is near or at the site where the viral polymerase initiates $26 \mathrm{~S}$ mRNA synthesis. The alphavirus RI population is cleaved into three RFs. The largest RF (20S in size) corresponds to a double-stranded form of the genome and represents intermediates active solely in genome synthesis. The other two RFs are derived from an RI active in subgenomic mRNA synthesis: The larger RF (18S in size) contains the double-stranded form of the sequence that is the $5^{\prime}$ two-thirds of the genome; the smaller RF ( $15 \mathrm{~S}$ in size) is the double-stranded form of the $3^{\prime}$ third of the genome that is the 26S mRNA sequence (Simmons and Strauss 1972). Therefore, we set out to determine the origin of the MHV subgenomic minus strands. We found (Sawicki and Sawicki 1990) that cells infected with MHV-A59 contained both genomic- and subgenomic-sized RIs, the latter of which would contain templates corresponding in length to the viral subgenomic mRNA. We also showed that the genome-length RI was not converted to a smaller or subgenome-length RF but only to the genome-length RF on treatment with RNase. This allowed us to conclude that coronaviruses produce subgenomic mRNAs using templates that are of similar size to each mRNA.

David Brian's laboratory (Sethna et al. 1991) demonstrated that the TGEV subgenomic minus strands contained a sequence complementary to the $5^{\prime}$ leader, and we confirmed this for the MHV-A59 subgenomic minus strands (Sawicki and Sawicki 1995). In light of these observations, two models could explain coronavirus transcription: the replicon model proposed originally by Sethna et al (1989) and our model (Sawicki and Sawicki 1995). With the replicon model, subgenomic mRNA would be made via leader-primed transcription using the fulllength minus strands as a template. Once made, these subgenomic mRNA would be replicated into minus strands that would in turn serve as templates for more subgenomic mRNA synthesis. Also, subgenomic mRNA that were virion associated (i.e., packaged into virions) could serve directly as templates for minus strand synthesis and result in the replication of the subgenomic mRNA. In our model (Sawicki and Sawicki 1995), which we called by the awkward name of "discontinuous extension of minus-strand RNA", the minus-strand templates for subgenomic mRNAs would be made directly from the genome and would utilize a discontinuous transcription process. We proposed that, during minus strand synthesis, the viral polymerase would pause at the IG sequences 
and then relocate to the $5^{\prime}$ end of the genome to finish transcription. This would result in the minus strand acquiring a sequence complementary to the $5^{\prime}$ leader sequence and activating it as a template for subgenomic mRNA synthesis. Those polymerases that failed to pause, or resumed elongation after pausing, would create the full- or genome-length minus strand template. The crucial observation that makes our model more attractive than the replicon model is that sequences downstream of the leader are required for replication signals. Neither transfected, subgenomic mRNA (Brian et al. 1994) nor subgenomic DI-RNA (Makino et al. 1991) replicated in cells infected with a helper virus. It appeared that sequences downstream of the leader RNA, 500 nt of the $5^{\prime}$ end for MHV-A59 that are present only in genomes and DI RNAs, are required and sufficient for viral RNA replication (Masters et al. 1994). Therefore, our model could account for and explain the known experimental observations.

Although the mechanism by which coronaviruses accomplish the synthesis of minus-strand templates for subgenomic mRNA synthesis are not known presently, it is clear that a working and testable model for coronavirus transcription must be able to explain or account for all the known facts. As Agnostino Bassi remarked "When Fact speaks, Reason is silent, because Reason is the child of Fact, not Fact the child of Reason." The known facts for coronavirus transcription include:

1. The formation of the $3^{\prime}$ coterminal nested set of subgenomic mRNA with a common leader sequence at their $5^{\prime}$ ends (discontinuous transcription).

2. The presence of transcriptionally active RIs that contain either genomelength or subgenome-length templates.

3. Polarity in the synthesis of subgenomic mRNAs. With a few exceptions, the relative synthesis of one subgenomic mRNA to another is determined by its distance from the $5^{\prime}$ end of the genomic minus strand, or the $3^{\prime}$ end of the genome. The closer the region encoding a subgenomic mRNA or subgenomic DI RNA is relative to the $5^{\prime}$ end of the genomic minus strand, the greater its synthesis level. Exceptions appear because of the IGS being too close to the $3^{\prime}$ end of the genome to allow polymerase to always bind stably.

4. The ratio of genome to subgenomic mRNA synthesis remains constant from beginning to end of the infectious cycle.

5. Mutations in the IGS affect the synthesis of subgenomic mRNA but not in a uniform and predictable way; some abolish subgenomic mRNA and some have little or no effect. 
6. A differential sensitivity to UV radiation proportional to the size of the synthesis of genomic and subgenomic mRNA, at least after subgenomic mRNA synthesis has started.

7. Synthesis of minus strands occurs simultaneously with the synthesis of plus strands. The only exception to date (An et al. 1998) may be due to the difficulty of the DI system chosen and very low numbers of viral molecules at early times p.i.

8. The capacity of subgenomic mRNA or a DI genome RNA or subgenomic DI RNA to acquire a leader RNA from a different genome (leader switching).

9. The high rate of recombination in coronaviruses.

The model of the discontinuous extension of the $3^{\prime}$ end of the minus strands has many attractive features that fit the experimental data. We believe it is a unifying model to probe the mechanism underlying the generation of subgenomic mRNA. It provides a useful framework to design experiments and interpret results.

Eric Snijder's lab has used an arterivirus, equine arteritis virus (EAV), that is also a member of the Nidovirales, to provide direct evidence for minus, not plus, strand synthesis being discontinuous (van Marle et al. 1999a). They changed the sequence in the IGS (in the plus orientation) or the TRS (in the minus orientation). They substituted one or both "C"s in the IGS UCAAC sequence at the end of the leader, in a body IGS (adjacent to a downstream gene), or in both. Changes in the leader IGS led to loss of all subgenomic mRNAs. In contrast, changes to the body IGS-7 led to loss of only mRNA-7. The very low amounts of mRNA-7 made under the latter conditions contained the mutated body IGS-7 sequence in their $5^{\prime}$ leader and not the original leader IGS sequence. Significantly, when both gene- 7 and the $5^{\prime}$ leader contained the same mutated sequence, full subgenomic mRNA-7 synthesis was restored. Thus, (1) complementary base pairing between TRS at the $3^{\prime}$ end of nascent minus strand "body" sequences and IGS on the end of the leader at the $5^{\prime}$ end of genome template appears necessary to obtain maximal subgenomic mRNA synthesis (i.e., formation of the templates for subgenomic mRNA). (2) It is unlikely that the recombination requires recognition of a specific sequence of IGS or TRS. Finally, (3) the discontinuous direction of synthesis was from body to leader and, thus, the discontinuous transcription step must happen during minus strand synthesis, that is, during the formation of subgenomic minus strands.

Presently, there is no experimental information on how this process occurs with coronaviruses. Only with arteriviruses has Eric Snijder's 
laboratory obtained direct information on a mechanism (van Marle et al. 1999a; Pasternak et al. 2003) and on the viral genes involved in subgenomic mRNA synthesis (van Marle et al. 1999b; van Dinten et al. 2000; Tijms and Snijder 2003). According to our model of $3^{\prime}$ discontinuous extension (Sawicki and Sawicki 1995), the viral polymerase would begin transcription at the $3^{\prime}$ end of the genome. It would pause after transcribing each of the IGS UCUAAAC elements. Each polymerase can then either elongate through the pause site, that is, continue transcription, or move to the $5^{\prime}$ end of the genome without copying the intervening sequences, that is, discontinuous transcription. The coronavirus polymerase might copy the template RNA in a fashion analogous to DNA-dependent RNA polymerases that retract at pause sites (Komissarova and Kashlev 1997a,b) and where the polymerase remains associated with the growing nascent strand rather than with the template. Of interest, a similar mechanism was recently proposed for proofreading by RNA polymerases, where the polymerase pauses, retracts, removes several nucleotides from the nascent $3^{\prime}$ end, and then resynthesizes this region (Shaevitz et al. 2003). Several gene products of ORF1b of coronaviruses and SARS CoV were identified that might function in such a proofreading mechanism, some of which are predicted to have nuclease activity (Snijder et al. 2003).

If retraction occurred when the coronavirus polymerase was transcribing the IGS, the exposed $3^{\prime}$ end of the nascent minus strand would relocate and align precisely to complementary sequences at the $3^{\prime}$ side of the leader RNA at the $5^{\prime}$ end of the genome and complete transcription of the subgenomic minus-strand RNA. The interaction of the nascent minus strand with the $5^{\prime}$ end of the genome might be mediated by protein:protein interactions between the polymerase attached to the growing minus strand and a protein associated with the $5^{\prime}$ end of the genome. This also could occur by the polymerase binding directly to the sequences downstream of the leader. The minus-sense or anti-IGS would align the polymerase at the end of the leader sequence in the genome template. If the $3^{\prime}$-terminal nucleotide of the anti-IGS matched, the polymerase would elongate and copy the leader sequence to put an antileader (complementary to the $5^{\prime}$ plus-strand leader sequence) on the $3^{\prime}$ end of the nascent, subgenomic minus strand. Thus the anti-IGS acts as a primer to complete transcription and copy the leader RNA. The IGS in the context of the sequences surrounding it would function as an attenuator element. The result of $3^{\prime}$ discontinuous extension during minus strand synthesis is that the subgenomic minus strands acquire the antileader sequence at their $3^{\prime}$ end. The replication complex that synthe- 
sized a subgenomic minus strand may retain it as a template and go on to synthesize subgenomic mRNA. The promoter for plus strand synthesis would be within the antileader sequence. As stated above, because sequences downstream of the leader are required for minus strand synthesis (Masters et al. 1994), only genomes can serve as templates for genomic and subgenomic minus strands; therefore, a great advantage of this strategy is that subgenomic mRNAs cannot be replicated and cannot behave as DI RNAs.

\section{3 \\ Kinetics of Plus- and Minus-Strand RNA Synthesis and Sensitivity to Translational Inhibition}

Our interest in coronavirus RNA synthesis initially came from a report in 1982 by Brayton et al (Brayton et al. 1982). They claimed that minus strand synthesis was temporally distinct from plus strand synthesis when MHV-infected cell extracts were assayed in vitro. They reported that extracts of A59-infected cells at $1 \mathrm{~h}$ p.i. made only minus strands and extracts of cells harvested at $6 \mathrm{~h}$ p.i. made only plus strands. Other plus-strand RNA viruses that replicate via RNA-dependent RNA polymerases show concurrent synthesis of minus and plus strands, unless minus strand synthesis ceases at some later time in infection [as is the case for alphaviruses (Sawicki and Sawicki 1998)]. Thus minus strand synthesis in the absence of plus strand synthesis would be a very unusual transcription pattern. We began a comparative analysis of minus and plus strand syntheses by MHV-A59 and the alphavirus Sindbis to probe the veracity of this observation and its implications for the regulation of transcription.

We first established optimal conditions for infection of $17 \mathrm{cl}-1$ cells. If infection was done in low-pH medium ( $\mathrm{pH}$ 6.8), infected cultures delayed the expression of the typical cytopathic effect and did not undergo extensive syncytium formation until after $10 \mathrm{~h}$ p.i., thus allowing us to examine a broader range of times after infection. Fusion of the monolayer at $\mathrm{pH}$ 7-7.5 normally resulted in the shutdown of viral replication much earlier and yielded only low titers of progeny. We found that MHV minus-strand RNA synthesis was detectable as early as $3 \mathrm{~h}$ p.i. in pulselabeling experiments using ${ }^{3} \mathrm{H}$-uridine in the presence of dactinomycin and that the minus strands were exclusively in the partially doublestranded RI population, as expected for template molecules. The rate of MHV minus strand synthesis peaked at 5-6 h p.i. at $37^{\circ} \mathrm{C}$ (or at about 
$10-12 \mathrm{~h}$ p.i. if infection was at $28^{\circ} \mathrm{C}-30^{\circ} \mathrm{C}$ ) and then declined to about $20 \%$ of its maximum rate but interestingly did not cease. Adding cycloheximide before $3 \mathrm{~h}$ p.i. prevented viral RNA synthesis; addition of the drug after RNA synthesis was ongoing led in its inhibition. Unlike alphavirus but similar to picornavirus replication, both MHV plus- and minus-strand polymerase activities were unstable but in MHV they differed in their turnover rates: Minus strand synthesis was almost immediately inhibited after translation inhibition, that is, addition of cycloheximide or puromycin, whereas both genome and subgenomic mRNA syntheses continued for 30-60 min before declining slowly. The short half-life of the coronavirus minus-strand polymerase activity may reflect a possible role in minus strand synthesis of intermediate or partially processed polyproteins, whose further processing leads to loss of this enzymatic activity. Whether loss of minus-strand polymerase activity is correlated with its conversion to another type of polymerase activity (plus strand synthesis) is not known. The latter has been demonstrated for alphaviruses (Lemm et al. 1994; Shirako and Strauss 1994; Wang et al. 1994).

\section{4}

\section{The Kinetics of Synthesis of the Subgenomic Minus Strands}

This was crucial to supporting or not supporting different models for coronavirus transcription. If, at all times, it was found that minus strand populations were composed of genome- and subgenomic-sized molecules in fixed but nonequal ratios to each other, then the $3^{\prime}$ discontinuous minus strand transcription model would be supported. Therefore, in an attempt to disprove our model, we used several approaches to ask which size minus-strand molecules first appeared and what the plus strand products were at that time. PCR technology allows the detection of very small numbers of target sequences. We designed specific primer sets to allow the amplification of genome (RNA 1) plus- or minus-strand sequences separately from the amplification of subgenome RNA 7 plusand minus-strand sequences. One of the primers in each set was targeted to the $5^{\prime}$ leader (or $3^{\prime}$ antileader) sequences at the ends of the RNAs and the other of each pair to the "body" sequence that would be a known distance downstream or upstream from the end. Our first studies used infected cell RNAs isolated at $7 \mathrm{~h}$ p.i., when viral transcription is readily detected by pulse-labeling in vivo (Sawicki and Sawicki 1995). The results indicated that at $7 \mathrm{~h}$ p.i. cells contained minus strands corre- 
sponding to both minus-sense RNA1 and RNA7 and that both of these minus-strand molecules contained an antileader on the $3^{\prime}$ ends. Uninfected cells did not amplify a PCR product with these primers, but cells infected with $\mathrm{MHV}$ in the cold $\left(4^{\circ} \mathrm{C}\right)$ and harvested at $4^{\circ} \mathrm{C}$ after removing unadsorbed virus contained only genome RNA and not the minusstrand copy of the genome. Nor did they contain plus- or minus-strand copies of RNA 7. Our twice isopycnic gradient-purified virion preparations contained small amounts of mRNA 7, but these were in different "particles" than the virions because they were not adsorbed to cells during the 1-h period at $4^{\circ} \mathrm{C}$. The results demonstrated directly that the subgenomic mRNAs and subgenomic minus-strand RNAs arise from genome plus strands because that was the only RNA in virions that initiated the infection (Sawicki and Sawicki 1995).

Our experiments consistently found that radiolabel was detectable in the subgenomic RI population as early as $1.5 \mathrm{~h}$ p.i. and at the same time that radiolabel was detectable in the genomic RI population. Because the genome RNA is 15-20 times larger than RNA 7, each molecule of $60 \mathrm{~S}$ RNA contains on average of 15-20 times more uridine residues and is thus "hotter" than a molecule of RNA 7 and easier to detect in pulse labels. Increased exposure of the gels to compensate for this imbalance verified the presence at each time period of the subgenomic RI population. Moreover, the relative proportion of each of the seven size classes of RI was maintained at all times studied, both early and late. We interpreted our results to indicate that both genome and subgenomic minus strands are produced at the earliest times in infection and thus must be derived from input genome plus strands. We have failed so far to detect a time early in infection when only genomic minus strands are made. Thus the synthesis of subgenomic mRNAs observed at 1.5-2 h p.i., or other early times, must arise from copying of complementary and subgenomic minus-strand templates.

\section{5}

\section{Subgenomic MHV RIs Exist in Infected Cells and Are Transcriptionally Active in mRNA 2-7 Synthesis}

Up until 1989, the prevailing model for coronavirus transcription and the formation of the $3^{\prime}$ nested set of mRNAs was "leader-primed transcription" (Baric et al. 1983). The first report that coronaviruses might use subgenomic templates came from the lab of David Brian (Sethna et al. 1989), who reported finding a $5^{\prime}$ nested set of minus strands in 
TGEV-infected cells by Northern blotting. Thus the number and kind of minus-strand RNAs matched those found for the virus's plus-strand RNAs. This exciting report led us to probe whether MHV A59-infected cells contained more than one size class of RF RNA and, if so, whether these were derived from genome-length RI molecules. Our results showed seven double-stranded RF cores in infected cell extracts digested with limited amounts of RNase and demonstrated that they were proportional in size and amounts to template the $3^{\prime}$ nested set of viral mRNAs and account for their nonequal ratios (Sawicki and Sawicki 1990). Thus the abundance of individual MHV mRNAs is a direct result of the number of their minus-strand templates. We also showed that this family of RF molecules did not come from an originally genome-length RI by demonstrating the existence of a family of RI molecules in the absence of RNase treatment. Finally, nascent labeling experiments using very short pulses with $900 \mu \mathrm{Ci}{ }^{3} \mathrm{H}$-uridine/ml demonstrated that the subgenomic RI RNA accumulated label first and only later was radiolabel chased into single-stranded mRNA (Sawicki and Sawicki 1990). This precursor-product relationship is what is expected for transcription templates, leading us to conclude that discontinuous transcription must occur during minus strand synthesis and its result was the $5^{\prime}$ nested set of minus-strand templates. If these contained an antileader sequence at their $3^{\prime}$ end and a poly $\mathrm{U}$ at their $5^{\prime}$ end, they could be directly transcribed into the subgenomic mRNAs. Thus the presence of subgenomic minus strands suggested a replication strategy that would be common to coronaviruses (and arteriviruses) and unique among the singlestranded RNA animal viruses. We suggested and favor the hypothesis that subgenomic minus strands are produced by discontinuous transcription directly from genome plus strands and are the only templates for subgenomic mRNAs.

\section{6}

\section{Characterization of Coronavirus Native RI/TIs and Native RF/TFs}

The replicative structures used by MHV to produce its genome and subgenomic mRNAs have the classic properties exhibited by viral RIs and RFs but are of genome or subgenomic length. Replicative intermediates are of genome length and have multiple replicases and nascent RNA chains. We proposed the term transcriptive intermediates (TI or TF) to refer to the same type of transcriptionally active coronavirus template structure but whose templates are of subgenome-length and whose 


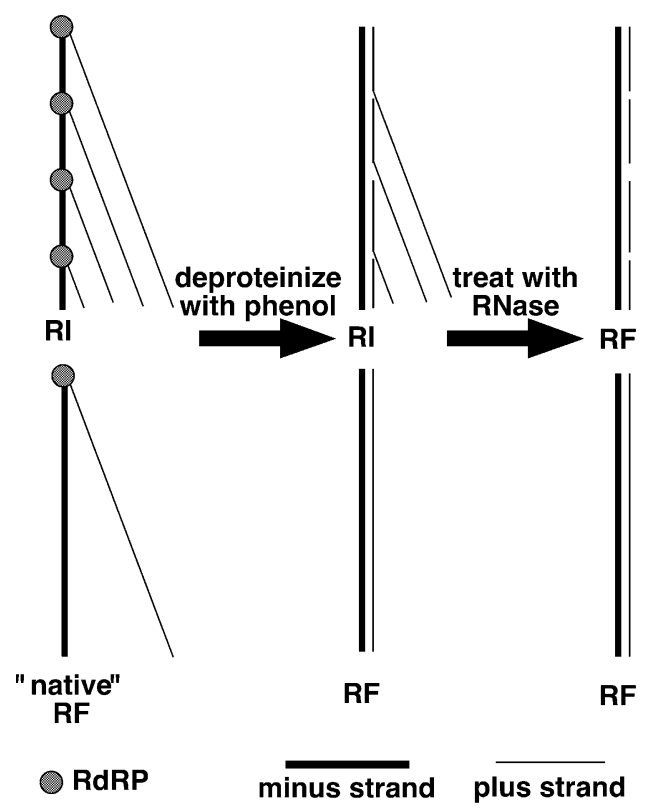

Fig. 3. Models for the structures of the coronavirus RI (TI for subgenomic templates) and RF (TF) structures. In infected cells, these molecules are predicted to have the "native" structures and to be essentially single stranded, with one or more RNA-dependent RNA polymerases elongating along the length of the template. When isolated from cells and deproteinized with phenol and chloroform (or proteases), the polymerase and other proteins are removed, allowing the nascent strands to collapse and bind to complementary sequences of the template. The RI/TI have multiple nascent strands and would be only partially double stranded and mostly single stranded; the RF/TF have only one or two nascent strands and would be almost or fully double stranded even without nuclease digestion. Finally, limited digestion of the deproteinized RNAs with RNase cleaves off all single-stranded RNA chains and converts these structures to fully double-stranded RF or TF molecules

product is one of the six size classes of subgenomic mRNA. Such transcriptionally active structures are likely essentially single stranded in vivo, with only small regions near the polymerase in double-stranded form. When proteins are removed from RI and TI structures, and the resulting RNA is digested with low concentrations of RNase, a doublestranded core or replicative form is recovered from each (also called a RF or TF). A schematic of these structures is shown in Fig. 3.

The RI/TI and RF/TF differ in their relative proportion of singlestranded and double-stranded character. Viral infections also form na- 
tive $\mathrm{RF}$ and $\mathrm{TF}$ under conditions when replicase or transcriptases are limited or initiation is reduced. Deproteinized native RF/TF would be completely or nearly completely double stranded as a result of having its one or few polymerases engaged in transcription and near the end of each template at the time of cell lysis. Thus plus and minus strands in native RF/TF would be mostly equal in length. Native RF are soluble in high-salt solutions $(2 \mathrm{M} \mathrm{LiCl}$ or $1 \mathrm{M} \mathrm{NaCl}$ ) and they function as transcription intermediates based on their rapid incorporation of radiolabeled nucleotide precursors. On the other hand, RI is a multistranded intermediate, whose multiple polymerases are associated with an equal number of nascent RNA chains of varying lengths. Its large percentage of single-strandedness makes the RI insoluble in high-salt solutions, allowing its physical separation from RF/TF, tRNA, and DNA. The MHV structures characterized so far are those active in plus strand synthesis (Sawicki et al. 2001). It is assumed that intermediates active in minus strand synthesis share some or all of these classic features, but these intermediates have not yet been isolated free of RI/TI active in plus strand synthesis.

Our analysis of RNA structures formed in MHV-infected cells found that, in addition to the RI and native RF, MHV-infected cells contained six species of RNA intermediates active in transcribing subgenomic mRNA (Sawicki et al. 2001). When radiolabeled between 5 and $6 \mathrm{~h}$ p.i., $70 \%$ of MHV replicating and transcribing structures in 17cl-1 cells had the physical properties of RI/TI and 30\% were native RF/TF. The seven size classes of intermediates or native forms can be separated partially from each other by velocity sedimentation on sucrose gradients or by gel filtration chromatography on Sepharose 2B and Sephacryl S-1000. The MHV RI/TI and native RF/TF double-stranded core structures are resistant to a range of RNase T1 concentrations (1-10 units/mg RNA) but are degraded during exposure to intermediate to high concentrations of pancreatic RNase (RNase A). There is an exposed poly(A) sequence on each of the size classes of TI and TF long enough to allow their adsorption to magnetic beads containing oligo(dT)25. It is likely that the genome-sized RF and RI also possess poly(A) sequences, but the large overall size of these molecules prevented their stable attachment to the beads. It was demonstrated directly that RI and TI are transcriptionally active structures in vivo, and thus biologically significant, from the finding that each size class of replicative molecule was able to rapidly incorporate radiolabeled precursors into nascent RNA chains (Fig. 4). 


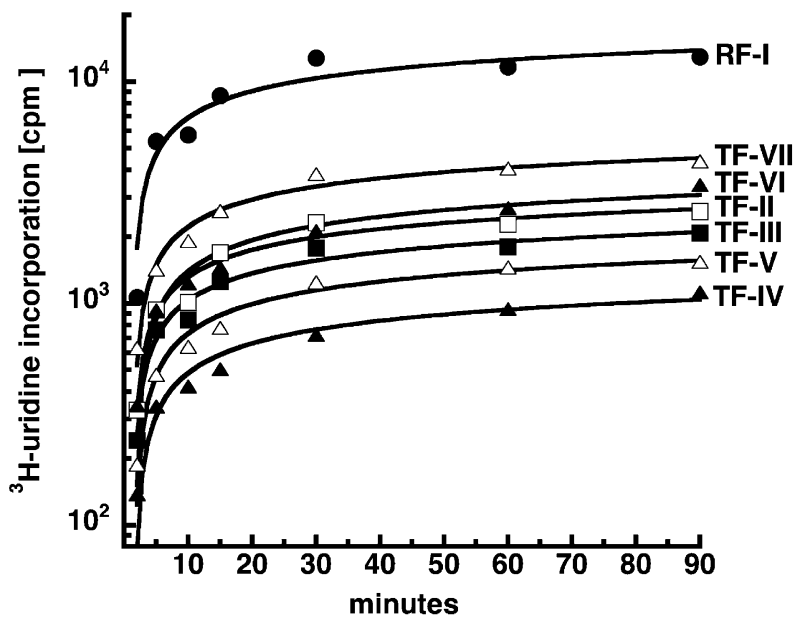

Fig. 4. The subgenome-length TI and native TF are transcriptionally active and incorporate radioactive nucleotide precursors at the same rate (kinetics) as the genome-length RI and native RF. See also Sawicki et al (2001). This evidence, which was also shown in our 1990 publication (Sawicki and Sawicki, 1990), dispelled any notion that the incorporation of ${ }^{3} \mathrm{H}$-uridine into RIs with subgenome-length templates is into dead-end products

In summary, the discontinuous transcription process occurs during minus strand synthesis and produces a $5^{\prime}$ nested set of minus strands each of which contains a copy of the antileader sequence at its $3^{\prime}$ end. This sequence likely functions or aids in polymerase initiation for synthesis of the subgenomic mRNA. This strategy has several key advantages: It enables the coronavirus (nidovirus) transcriptase to skip over sequences in the template that are not needed in a particular subgenomic mRNA, thus conserving cellular substrates. Perhaps more importantly, it allowed the evolution of the "nested set" strategy, whereby each open reading frame is expressed at the $5^{\prime}$ end of a specific mRNA. This in turn allows for regulation at the transcription level. The number of minusstrand templates controls the number of each mRNA species, which controls the amount of its specific protein product(s). It is not surprising to find that mRNA 7 is produced in highest abundance and it encodes the viral capsid protein. This strategy also would enable the genome to encode essential regulatory sequences once, conserving linear genome space, and at the same time to provide for these sequences to be added to each of the resulting RNA species that require them to function efficiently (ribosome binding; polymerase initiation). 


\section{7}

\section{Turnover of MHV Replicative/Transcriptive Intermediates}

Coronavirus RI/TI and RF/TF are short-lived and turn over during infection and after exposure of infected cells to temperatures of $0^{\circ} \mathrm{C}-4^{\circ} \mathrm{C}$. We were the first to show that inhibition of protein synthesis in cells infected with MHV-A59 caused minus strand synthesis to stop almost immediately (Sawicki and Sawicki, 1986). Plus strand synthesis was also inhibited when translation was prevented, but its loss occurred more slowly over time. This, as we pointed out, was different from alphaviruses that make a very stable transcription complex that functions for many hours in the absence of new protein synthesis. The unstable nature of the coronavirus transcription complex has now also been shown for the arteriviruses (Den Boon et al. 1995; Snijder and Meulenberg, 1998). Recently we discovered (Wang and Sawicki 2001) that the minus-strand templates but not the plus strands made in MHV-infected cells are unstable and turn over. In most plus-strand viruses the minus strands are stable and are found not as free, single-stranded RNA but as components of RI or RF structures. Therefore, we were surprised to find that the RI/TI and RF/TF that had already accumulated in infected cells between 1-6 h p.i. disappeared over time after $6 \mathrm{~h}$ p.i. Minus strands made after $6 \mathrm{~h}$ p.i. accumulated temporarily and replaced some of the minus strands synthesized earlier. Treatment with cycloheximide for extended periods at early or late times p.i. led to the loss of $90 \%$ or more of the pre-existing native RF/TFs and RI/TIs and prevented the synthesis of new ones. The amounts remaining at each time subsequent to drug addition were proportional to the reduced rates of plus strand synthesis observed. Removal of the drug led to a burst of minus strand synthesis and full recovery of transcription. The turnover of RI/TI and RF/TF was specific to MHV and did not affect the RI/RFs of alphaviruses (Sindbis virus) in cells coinfected with both viruses, as shown in Fig. 5.

Thus MHV RI/TIs function for a limited time, whereas the Sindbis RI is a stable replication/transcription complex. The MHV turnover process involved release of minus strands, possibly with dissociation of the entire replication/transcription complex, after which these minus strands were degraded. Minus strands not associated with plus strands were present in infected cells late in infection and after cycloheximide treatment. These "free" minus strands could be captured as RNase-resistant, double-stranded RNA if they were allowed to hybridize with the excess plus strands in the cell lysates before RNase treatment. The results suggest that the continuation of minus strand synthesis is essential for viral 


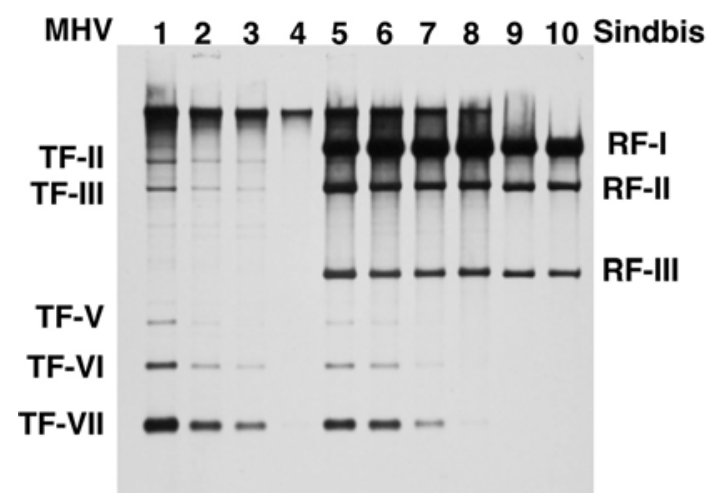

Fig. 5. MHV replicative and transcriptive intermediates are unstable and turn over throughout infection, in contrast to alphavirus (Sindbis, strain HR) intermediates that are stable once formed. Cultures of $17 \mathrm{cl}-1$ cells were infected (at an MOI of 100) with MHV A59 (lanes 1, 2, 3, and 4), with MHV + Sindbis (lanes 5, 6, 7, and 8), or with Sindbis alone (lanes 9 and 10). The infected cells were labeled with ${ }^{3} \mathrm{H}$-uridine from 3 to $7 \mathrm{~h}$ p.i. (lanes 1 and 5), from 3 to $9 \mathrm{~h}$ p.i. (lanes 2 and 6), from 3 to $11 \mathrm{~h} \mathrm{p.i.}$ (lanes 3, 7, and 9), or from 3 to $13 \mathrm{~h}$ p.i. (lanes 4, 8, and 10). The deproteinized and RNase-treated RF/TF cores of the intermediates and native forms were obtained and electrophoresed on a $1 \%$ agarose-TBE gel. The gel was prepared for fluorography

transcription seen late in infection and that the coronavirus minusstrand templates need to be continuously replaced. Failure to continue minus strand synthesis would cure the infection. Thus we would argue that the persistence of MHV infections would be dependent on host cell environments that either allow the virus to continue to form new polymerases for minus strand synthesis or block the turnover of previously made template complexes. Although this turnover resembles that seen for poliovirus RI being converted to an inactive RF, release of minus strands from RI/RF and TI/TF RNA is unique to coronaviruses (and probably arteriviruses) among animal RNA viruses. Somewhat surprisingly, it resembles what is known for phage $\mathrm{Q} \beta$ transcription, where the template and product strands are released as single strands after the polymerase has copied the template (Dobkin et al. 1979).

Even more unexpected was our finding (Wang T. and Sawicki, unpublished results) that exposure to cold $\left(0^{\circ} \mathrm{C}-4^{\circ} \mathrm{C}\right.$ for as little as $\left.20 \mathrm{~min}\right)$ led to the disappearance of RI/RF and TI/TF RNA. Viral double-stranded RNA that had been present in cells labeled with ${ }^{3} \mathrm{H}$-uridine for several hours or even $30 \mathrm{~min}$ before exposure to cold was no longer detectable. We could recover RI/RF and TI/TF RNA by returning the cells to $37^{\circ} \mathrm{C}$. 
However, if we added cycloheximide when we returned the cells to $37^{\circ} \mathrm{C}$, viral RNA synthesis failed to recover, suggesting that viral polymerase proteins were needed. Recovery of viral RNA synthesis was preceded by a burst of minus strand synthesis, and these new products were utilized as templates for plus strand synthesis. Rapid loss of RI/RF and TI/TF was also found after infected cells were treated with trypsin or were scraped from the surface of the culture dish at $0^{\circ} \mathrm{C}, 25^{\circ} \mathrm{C}$, and $37^{\circ} \mathrm{C}$. The interpretation of these observations is difficult at this time, but they may indicate that the replicase-transcriptase is associated with the cytoskeleton and this association is disrupted under conditions that alter cell shape or cold-sensitive protein interactions.

\section{8}

\section{A Working Model}

Figure 6 presents our working model for coronavirus replication and transcription. The infecting genome is translated into ppla and pplab. Within $1 \mathrm{~h}$ of infecting the cells we can detect genome and subgenomic mRNA being made. As far as we can determine, both genomic and subgenomic minus strands are made very early after the genome enters the cell. We believe that parts of the pplab function, at least initially, as uncleaved or partially cleaved polyprotein forms to make minus strands. Once the minus strands are made they would be rapidly converted to templates for use in plus strand synthesis. There does not appear to be any difference early or late in the ratio of genome and subgenomic mRNA; it remains fixed from the earliest moments at which the synthesis of viral RNA can be detected. As we published many years ago (Sawicki and Sawicki 1986), minus strands are made throughout infection although their synthesis declines after 6-7 h p.i., but so does the amount of plus strand synthesis. Only the genomes are capable of serving as templates for minus strand synthesis because only they have a replication signal at their $5^{\prime}$ and $3^{\prime}$ ends. The subgenomic mRNA cannot serve as templates for minus strand synthesis because they lack the $5^{\prime}$ replication signal. After the minus strands serve as templates for several rounds of plus strand synthesis, they are released from the replication/transcription complexes, which do not appear to be different for those making genomes compared with those making subgenomic mRNA. After their release they are subject to degradation by a RNase activity of unknown origin that seems to be specific for them because the genome and subgenomic mRNA are stable, perhaps because they are engaged with the ribo- 


\section{Model for Coronavirus Replication and Transcription}

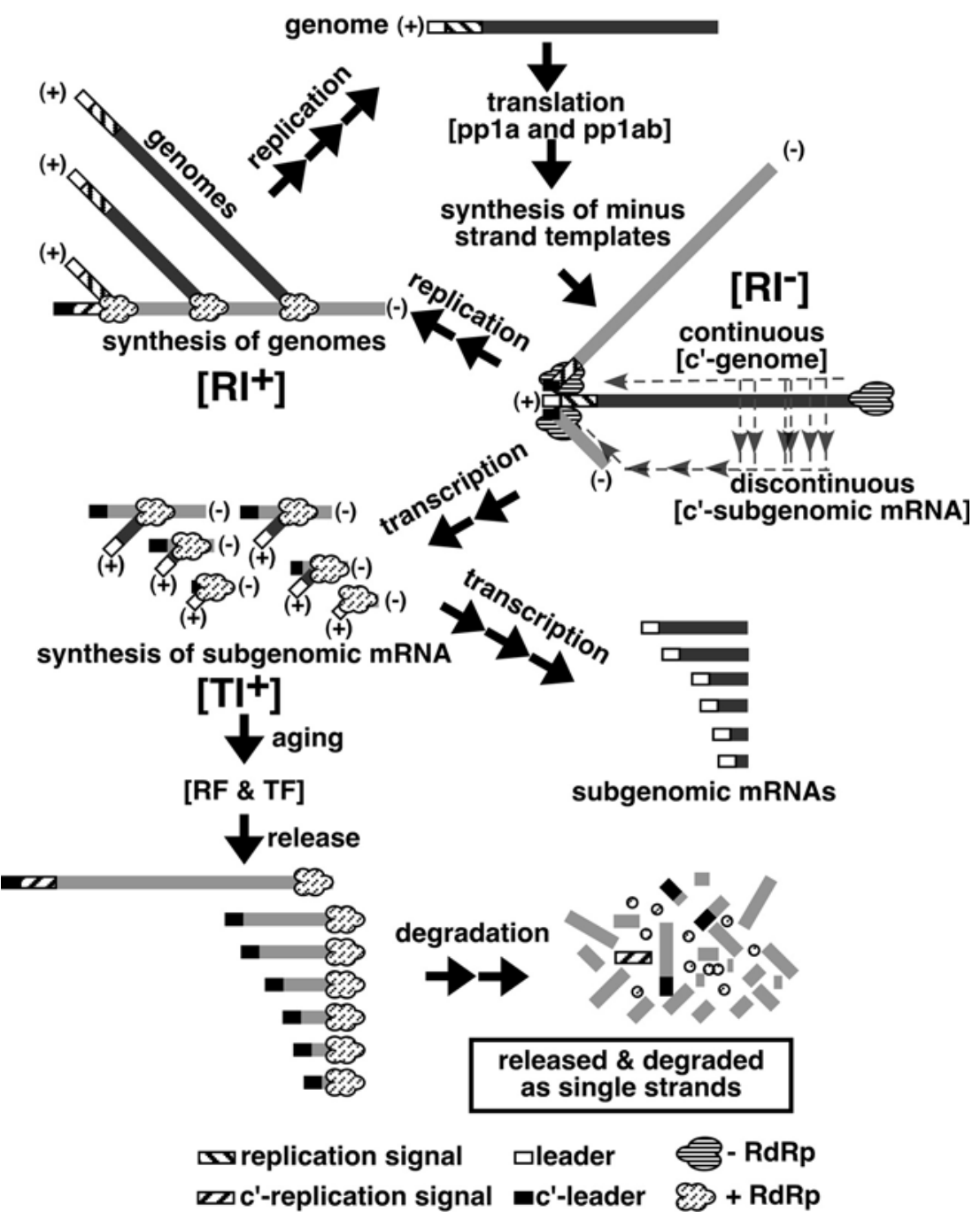

Fig. 6. Depiction of our model for the replication and transcription strategy of coronaviruses 
some or in the process of being encapsidated by $\mathrm{N}$ proteins that act to protect them. With MHV-A59 cell fusion starts about the time viral RNA synthesis reaches a maximum rate and cell fusion results in early killing of the cells. With mutants we have isolated that do not cause cell fusion, the cells survive until $24 \mathrm{~h}$ after infection even though the virus was released by $8-10 \mathrm{~h}$ p.i. We can delay the cell fusion and cell death with MHV-A59 if we use medium that has a pH below 6.5 and if we lower the temperature to $33^{\circ} \mathrm{C}$. If we keep the cells alive, the loss of RI/TI is easily observable. We speculate that coronaviruses encode in ppla or pplab an RNase, for example, the XendoU in nsp15 perhaps (Snijder, Bredenbeek et al. 2003), that is responsible for the degradation of the minus strands. Our hypothesis, or working model, is that the replication/transcription complex ages after its synthesis: First it has minus-strand activity, then it has plus-strand activity, and then it further ages and degrades the template it is using to make plus strands. Aging may be through a proteolytic cleavage pathway by the $3 \mathrm{CL}^{\mathrm{PRO}}$ in $\mathrm{nsp} 5$, and the final cleavages release or activate the RNase activity of the complex. This working model provides a framework for our future studies of coronavirus transcription and replication. Important for this effort will be the examination of $t s$ RNA-negative mutants and designing mutants with reverse genetics.

Acknowledgements We wish to acknowledge support from the National Institutes of Health (SGS, AI-28506). We especially wish to acknowledge the contributions to this work of colleagues and friends, whose intellectual input and challenges over the years have assisted our efforts. In particular, we wish to thank Larry Sturman for starting us on this exciting research path.

\section{References}

An S, Maeda A, Makino S (1998) Coronavirus transcription early in infection. J Virol 72:8517-8524

Baric RS, Stohlman SA, Lai MMC (1983) Characterization of replicative intermediate RNA of mouse hepatitis virus: presence of leader RNA sequences on nascent chains. J Virol 48:633-640

Brayton PR, Lai MMC, Patton CD, Stohlman S (1982) Characterization of two RNA polymerase activities induced by mouse hepatitis virus. J. Virol 42:847-853

Brian DA, Chang RY, Hofmann MA, Sethna PB (1994) Role of subgenomic minusstrand RNA in coronavirus replication. Arch Virol Suppl 9:173-180

Brockway SM, Clay CT, Denison MR (2003) Characterization of the expression, intracellular localization and replication complex association of the putative mouse hepatitis virus RNA-dependent RNA polymerase. J Virol 77:10515-10527 
Cavanagh D (1997) Nidovirales: a new order comprising Coronaviridae and Arteriviridae. Arch Virol 142:629-633

David-Ferreira JF, Manaker RA (1965) An electron microscope study of the development of a mouse hepatitis virus in tissue culture cells. J Cell Biol 24:57-64

Den Boon JA, Spaan WJ, Snijder EJ (1995) Equine arteritis virus subgenomic RNA transcription: UV inactivation and translation inhibition studies. Virology 213:364-372

Dobkin C, Mills DR, Kramer FR, Spiegelman S (1979). RNA replication: required intermediates and the dissociation of template, product and the Qbeta replicase. Biochemistry 18:2038-2044

Jacobs L, Spaan WJ, Horzinek MC, van der Zeijst BA (1981) Synthesis of subgenomic mRNAs of mouse hepatitis virus is initiated independently: evidence from UV transcription mapping. J Virol 39:401-406

Komissaarova N, Kashlev M (1997a) RNA polymerase switches between inactivated and activated states by translocating back and forth along the DNA and the RNA. J Biol Chem 272:15329-15338

Komissaarova N, Kashlev M (1997b) Transcriptional arrest: Escherichia coli RNA polymerase translocates backward, leaving the $3^{\prime}$ end of the RNA intact and extruded. Proc Natl Acad Sci USA 94:1755-1760

Kuo L, Master P (2003) The small envelope protein E is not essential for mouse coronavirus replication. J Virol 77:4597-4608

Lai MMC, Patton CD, Baric RS, Stohlman SA (1983) Presence of leader sequences in the mRNA of mouse hepatitis virus. J Virol 46:1027-1033

Lai MMC, Patton CD, Stohlman SA (1982) Replication of mouse hepatitis virus: negative strand RNA and replicative form RNA are of genome length. J Virol 44:487-492

Lai MMC, Baric RS, Brayton PR, Stohlman SA (1984) Characterization of leader RNA sequences on the virion and mRNAs of mouse hepatitis virus, a cytoplasmic RNA virus. Proc Natl Acad Sci USA 81:3626-3630

Lai MMC, Cavanagh D (1997) The molecular biology of coronaviruses. Adv Virus Res 48:1-100

Lai MMC, Holmes KV (2001) Coronaviridae: The Viruses and Their Replication. In Fields BN, Knipe DM, Howley PM and Griffin DE (eds). Field's Virology, $4^{\text {th }}$ Edition, volume 1, Lippincott, Williams and Wilkins, Philadelphia.

Lemm JA, Rumenapf T, Strauss EG, Strauss JH, Rice CM (1994) Polypeptide requirements for assembly of functional Sindbis virus replication complexes: a model for temporal regulation of minus strand and plus strand RNA synthesis. EMBO J 13:2925-2934

Makino S, Joo M and Makino JK (1991) A system for study of coronavirus mRNA synthesis: a regulated, expressed subgenomic defective interfering RNA results from intergenic site insertion. J Virol 65:6031-6041

Manaker RA, Piczak CV, Miller AA, Stanton MF (1961) A hepatitis virus complicating studies with mouse leukemia. Natl Cancer Inst 27:29

Masters PS, Koetzner CA, Kerr CA, Heo Y (1994) Optimization of targeted RNA recombination and mapping of novel nucleocapsid gene mutations in the coronavirus mouse hepatitis virus. J Virol 68:328-337 
Pasternak AO, van den Born E, Spaan WJ, Snijder EJ (2003) The stability of the duplex between sense and antisense transcription-regulating sequences is a crucial factor in arterivirus subgenomic mRNA synthesis. J Virol 77:1175-1183

Sawicki DL, Sawicki SG (1998) Role of the nonstructural polyproteins in alphavirus RNA synthesis. Adv Exp Med Biol 440:187-198

Sawicki SG, Sawicki DL (1986) Coronavirus minus strand synthesis and effect of cycloheximide on coronavirus RNA synthesis. J Virol 57:328-334

Sawicki SG, Sawicki DL (1990) Coronavirus transcription: subgenomic mouse hepatitis virus replicative intermediates function in RNA synthesis. J Virol 64:10501056

Sawicki SG, Sawicki DL (1995) Coronaviruses use discontinuous extension for synthesis of subgenome-length negative strands. Adv Exp Med Biol 380:499-506

Sawicki DL, Wang T, Sawicki SG (2001) The RNA structures engaged in replication and transcription of the A59 strain of mouse hepatitis virus. J Gen Virol 82:385396

Sethna PB, Hofmann MA, Brian DA (1991) Minus-strand copies of replicating coronavirus mRNAs contain antileaders. J. Virol 65:320-325

Sethna PB, Hung SL, Brian DA (1989) Coronavirus subgenomic minus strand RNAs and the potential for mRNA replicons. Proc Natl Acad Sci USA 86:5626-5630

Shaevitz JW, Abbondanzieri EA, Landick R, Block SM (2003) Backtracking by single RNA polymerase molecules observed at near-base-pair resolution. Nature 426:684-687

Shirako Y, Strauss JH (1994) Regulation of Sindbis virus RNA replication: uncleaved P123 and nsP4 function in minus strand RNA synthesis, whereas cleaved products from P123 are required for efficient plus strand synthesis. J Virol 68:18741885

Siddell SG (1995) The Coronaviridae. Plenum Press, New York.

Simmons DT, Strauss JH (1972) Replication of Sindbis virus II. Multiple forms of double-stranded RNA isolated from infected cells. J Mol Biol 71:615-631

Snijder EJ, Bredenbeek PJ, Dobbe JC, Thiel V, Ziehbuhr J, Poon LL, Guan Y, Rozanov M, Spaan WJ, Gorbalenya AE (2003) Unique and conserved features of genome and proteome of SARS-coronavirus, an early split-off from the coronavirus group 2 lineage. J Mol Biol 331:991-1004

Snijder EJ, Meulenberg JJ (1998) The molecular biology of arteriviruses. J Gen Virol 79:961-979

Spaan WJ, Delius H, Skinner M, Armstrong J, Rottier P, Smeekens S, van der Zeijst BA, Siddell SG (1983) Coronavirus mRNA synthesis involves fusion of non-contiguous sequences. EMBO J 2:1839-1844

Stern DF, Sefton BM (1982) Synthesis of coronavirus mRNAs: kinetics of inactivation of infectious bronchititis virus RNA synthesis by UV light. J Virol 42:755-759

Sturman, LS, Takemoto KK (1972) Enhanced growth of a murine coronavirus in transformed mouse cells. Infect Immun 6:501-507

Thiel V, Herold J, Schelle B, Siddell S (2001a) Infectious RNA transcribed in vitro from a cDNA copy of the human coronavirus genome cloned in vaccinia virus. J Gen Virol 82:1273-1281

Thiel V, Herold J, Schelle B, Siddell S. (2001b) Virus replicase gene products suffice for coronavirus discontinuous transcription. J Virol 75:6676-6681 
Tijms MA, Snijder EJ (2003) Equine arteritis virus non-structural protein 1, an essential factor for viral subgenomic mRNA synthesis, interacts with the cellular transcription co-factor p100. J Gen Virol 84:2317-2322

van der Most RG, deGroot RJ, Spaan WJ (1994) Subgenomic RNA synthesis directed by a synthetic defective interfering RNA of mouse hepatitis virus: a study of coronavirus transcription initiation. J Virol 68:3656-3666

van Dinten LC, van Tol H, Gorbalenya AE, Snijder EJ (2000) The predicted metal binding region of the arterivirus helicase protein is involved in subgenomic mRNA synthesis, genome replication and virion biogenesis. J Virol 74:5213-5223

van Marle G, Dobbe JC, Gultyaev AP, Luytjes W, Spaan WJ, Snijder EJ (1999a) Arterivirus discontinuous mRNA transcription is guided by base pairing between sense and antisense transcription-regulating sequences. Proc Natl Acad Sci USA 96:12056-12061

van Marle G, Luytjes W, van der Most RG, van der Straaten T, Spaan, WJ (1995) Regulation of coronavirus mRNA transcription. J Virol 69:7851-7856

van Marle G, van Dinten LC, Spaan WJ, Lyuytjes W, Snijder EJ (1999b) Characterization of an equine arteritis virus replicase mutant defective in subgenomic mRNA synthesis. J Virol 73:5274-5281

Wang T, Sawicki SG (2001) Mouse hepatitis virus minus strand templates are unstable and turnover during viral replication. Adv Exp Med Biol 494:491-497

Wang YF, Sawicki SG, Sawicki DL (1994) Alphavirus nsP3 functions to form replication complexes transcribing negative strand RNA. J Virol 68:6466-6475 